\title{
Interaction of arbekacin with dialysis membrane
}

\author{
Motoki Urata', Yuki Narita', Daisuke Kadowaki', Kazutaka Tanoue², Issei Tashiro³, Masaki Fukunaga', \\ Akitomo Shibata ${ }^{4}$, Yutaka Yoshida ${ }^{5}$ Kazuaki Soejima ${ }^{6}$ and Sumio Hirata ${ }^{*^{*}}$
}

\begin{abstract}
Background: The interaction between arbekacin and the hemodialysis membrane is unclear. The aim of this study was to evaluate the adsorption of arbekacin (ABK) onto hemodialysis membranes and to establish a simpler system for evaluating the adsorption properties of the drug.

Methods: First, small cut pieces of polysulfone or sulfonated polyacrylonitrile (AN69) hollow fiber membranes were stirred in a solution of ABK for the qualitative assessment. Then, we designed the experimental system that was approximately 1/100 of the actual size using a small dialyzer "mini-module" of our original design for the polysulfone or AN69. We circulated ABK solution in this system for the quantitative assessment. Finally, we administered ABK in subjects undergoing hemodialysis as a clinical trial to evaluate the adsorption of ABK onto polysulfone or AN69.

Results: In the qualitative assessment, the rates of ABK adsorption onto polysulfone and AN69 were $6.2 \pm 2.9$ and $49.8 \pm 1.8 \%$, respectively. In the quantitative assessment, although there was almost no change in the circulating ABK concentration with the use of polysulfone, there was about $68 \%$ decrease in the circulating ABK concentration with the use of AN69. In the clinical trial, clearance by the dialyzer using AN69 was high at 10 min after starting dialysis, despite the sieving coefficient being 0 . However, reduction of ABK clearance was observed with time; the removal rate of $A B K$ at the completion of the dialysis using polysulfone or AN69 was about $67 \%$, with no difference between the two membranes. The main limitation of this study was the small sample size in the clinical trial.
\end{abstract}

Conclusions: These findings suggest that ABK is adsorbed onto AN69. Furthermore, the present adsorption experiment with a mini-module was considered useful as an evaluation system because it was easy to handle, using less solvent and drugs compared with a previous report, and it reflected the results of the clinical trial.

Keywords: Arbekacin, Dialysis membrane, Adsorption, Sulfonated polyacrylonitrile membrane

\section{Background}

Arbekacin is an effective aminoglycoside antibiotic against methicillin-resistant Staphylococcus aureus (MRSA) and is widely used in Japan to treat MRSA infection [1]. As arbekacin has a narrow therapeutic range, therapeutic drug monitoring (TDM) is considered necessary to ensure its effectiveness and safety. Japanese TDM guidelines recommend that the peak serum concentration of arbekacin be kept within the range of 15 to $20 \mu \mathrm{g} / \mathrm{mL}$ because arbekacin has a strong therapeutic effect when the ratio of

\footnotetext{
* Correspondence: hirata@kumamoto-u.ac.jp

${ }^{1}$ Division of Clinical Pharmacology, Center for Clinical Pharmaceutical

Sciences, Faculty of Pharmaceutical Sciences, Kumamoto University, 5-1

Oe-Honmachi, Chuo-ku, Kumamoto 862-0973, Japan

Full list of author information is available at the end of the article
}

the maximum blood concentration to the minimum inhibitory concentration is $8: 1$ or higher. Furthermore, as a high trough concentration increases the risk of kidney damage, it is recommended that the trough concentration be kept at $2 \mu \mathrm{g} / \mathrm{mL}$ or lower [2]. It is believed that between 70 and $90 \%$ of arbekacin is excreted through the urine unmetabolized [3-5], so it is necessary to determine dosages with kidney function in mind. In addition, as arbekacin is removed through hemodialysis, this must also be taken into account when determining dosages [5, 6].

Removal of substances through hemodialysis typically occurs by diffusion or filtration. Moreover, low molecular weight proteins such as $\beta_{2}$ microglobulin are removed by 
adsorption onto some hemodialysis membranes [7]. Adsorption of drugs such as the aminoglycoside antibiotic amikacin onto certain hemodialysis membranes has also been reported [8-10], so adsorption onto hemodialysis membranes is one mechanism of drug removal. In a previous in vitro experiment that we conducted, we found that arbekacin is adsorbed onto sulfonated polyacrylonitrile (AN69), a type of hemodialysis membrane [11]. However, it is unknown whether arbekacin is removed by adsorption in clinical practice. Moreover, the in vitro experimental system that we used in our previous study is complicated because it is at $1 / 3$ scale and requires a large volume of solvent and drug. The objective of this study was to assess the absorption properties of arbekacin onto hemodialysis membranes and to establish a simple system for assessing the adsorption properties of drugs.

\section{Methods}

\section{Qualitative assessment of adsorption using cut} hemodialysis membranes

The hemodialysis membranes used were polysulfone (APS-SA; Asahi Kasei Medical Co., Ltd., Tokyo, Japan) and AN69 (FILTRAL12; Baxter Co., Ltd., Tokyo, Japan). The housing was removed from the dialyzer of each hemodialysis membrane, and the hollow fibers were taken out and washed with $\mathrm{ddH}_{2} \mathrm{O}$. Subsequently, the hollow fibers were cut at $5-\mathrm{mm}$ intervals using a razor blade that had been washed with ethanol to remove the paraffin. Arbekacin (Habekacin Injection 100 mg; Meiji Seika Pharma Co., Ltd., Tokyo, Japan) was diluted with $50 \mathrm{mM}$ phosphoric acid ( $\mathrm{pH} 7.4$ ) to a concentration of $20 \mu \mathrm{g} / \mathrm{mL}$. Hollow fiber fragments (from a $57.5-\mathrm{cm}^{2}$ membrane surface area) were added to arbekacin solution $(50 \mathrm{~mL})$ and were agitated for $30 \mathrm{~min}$ at $37^{\circ} \mathrm{C}$ (Fig. 1a). A control run was performed using the same procedures without adding the hollow fiber fragments, and arbekacin solution was collected before and after the start of the experiment. The rate of adsorption was calculated using the following equation.

$$
M_{\mathrm{ads}}=\left(C_{\mathrm{con}}-C_{\mathrm{mem}}\right) / C_{\mathrm{con}} \times 100
$$

$M_{\text {ads }}$ is the rate of adsorption (\%), $C_{\text {con }}$ is the control concentration $(\mu \mathrm{g} / \mathrm{mL})$, and $C_{\text {mem }}$ is the concentration after addition of hemodialysis membrane and $30 \mathrm{~min}$ of agitation $(\mu \mathrm{g} / \mathrm{mL})$.

The experimental system was designed to be approximately $1 / 200$ of actual size (i.e., $10 \mathrm{~L}$ of extracellular fluid and a membrane surface area of $1.15 \mathrm{~m}^{2}$ ). The above experiment was run in triplicate.

\section{Quantitative assessment of in vitro adsorption using a mini-module}

The housing was removed from polysulfone and AN69 dialyzers, and the hollow fibers were taken out. Fibers from a membrane surface area of $115 \mathrm{~cm}^{2}$ were measured out. There were 70 hollow fibers from the polysulfone membrane and 82 from the AN69 membrane. These hollow fibers were put through an acrylic pipe connected to the dialysate port (internal diameter, $7 \mathrm{~mm}$; external diameter, $10 \mathrm{~mm}$; length, $15 \mathrm{~cm}$ ); after which, both ends were secured with an epoxy resin adhesive (Quick5; Konishi Co., Ltd., Osaka, Japan). Subsequently, both ends were covered with a blood port cap and were secured with adhesive to create a mini-module. To ensure that the hollow fibers were not damaged and there were no leaks from the connections, the dialysate port was plugged and air was sucked out of the opposite port using a syringe. Arbekacin was diluted with $50 \mathrm{mM}$ phosphoric acid $(\mathrm{pH} 7.4)$ to a concentration of $20 \mu \mathrm{g} / \mathrm{mL}$. The mini-module was connected to a peristaltic pump and $50 \mathrm{mM}$ phosphoric acid ( $\mathrm{pH} 7.4$ ) was circulated through it at $2 \mathrm{~mL} / \mathrm{min}$ for $30 \mathrm{~min}$ to clean it. Subsequently, arbekacin solution $(100 \mathrm{~mL})$ was circulated a) Experimental device

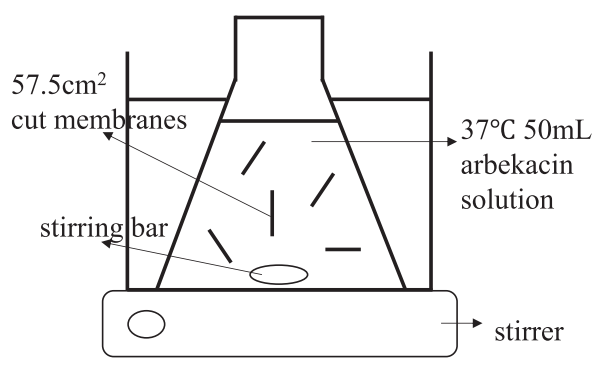

b) Adsorption rate of arbekacin

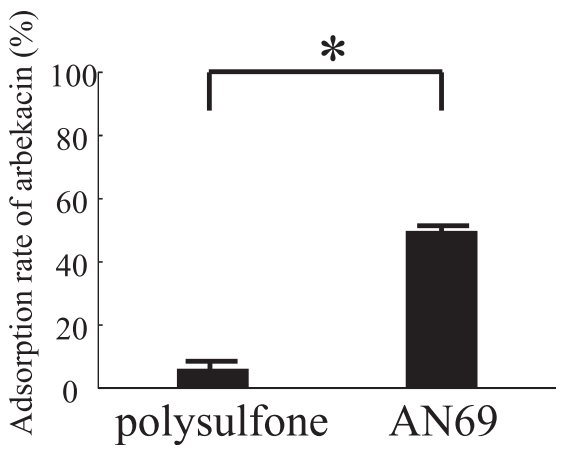

Fig. 1 Qualitative assessment of interaction of arbekacin with dialysis membranes. a Experimental device. $\mathbf{b}$ Adsorption rate of arbekacin. Results shown are mean \pm standard deviation $(n=3)$. ${ }^{P}<0.05$, compared with AN69. AN69 sulfonated polyacrylonitrile 
through the circuit for $240 \mathrm{~min}$ at $37{ }^{\circ} \mathrm{C}$. At that time, dialysate was not used, the flow rate of the arbekacin solution was $2 \mathrm{~mL} / \mathrm{min}$, and the flow rate of the filtrate was $0.6 \mathrm{~mL} / \mathrm{min}$. The filtrate obtained through ultrafiltration was circulated through again as arbekacin solution (Fig. 2a). With this method, the decrease in the concentration of the circulating arbekacin solution is believed to reflect the adsorption of arbekacin onto the hemodialysis membrane [12]. The arbekacin solution and the filtrate were collected in $200-\mu \mathrm{L}$ samples. Samples were collected at the start of circulation and 60, 120, and $240 \mathrm{~min}$ (i.e., the end of circulation) later when polysulfone was used and at the start of circulation and 30,60, 90, 120, 150, 180 , and $240 \mathrm{~min}$ (i.e., the end of circulation) later when AN69 was used. When samples were collected, arbekacin solution at the original concentration was added to replenish the collected amount. The experimental system was designed to be approximately $1 / 100$ of actual size (i.e., $10 \mathrm{~L}$ of extracellular fluid, a membrane surface area of $1.15 \mathrm{~m}^{2}$, and a blood flow rate of $200 \mathrm{~mL} / \mathrm{min}$ ). The above experiment was run in triplicate.

\section{Clinical study}

\section{Subjects}

The subjects were patients with MRSA infection who were at least 20 years old and on maintenance hemodialysis at the Blood Purification Center of Saiseikai Kumamoto Hospital between November 2010 and September 2014. Patients using an angiotensin-converting enzyme inhibitor, patients with a history of hypersensitivity to aminoglycoside antibiotics, patients with a family history of hearing impairment related to aminoglycoside antibiotics or any other kind of hearing impairment, and patients in whom proper evaluation and adequate follow-up were deemed by the study doctor to be impossible were excluded from the study.

2. Research ethics

This clinical study was approved by the Clinical Research and Medical Technology Ethics Committee of Kumamoto University (Advanced Medical Care No. 1111) and the ethics committee of Saiseikai Kumamoto Hospital, and was conducted in accordance with the Declaration of Helsinki. It was conducted in collaboration with doctors at the Blood Purification Center of Saiseikai Kumamoto Hospital after obtaining freely given written informed consent from subjects. To fully ensure patients' privacy, data were anonymized so that patients could not be identified by their names or other personal information.

3. Drug administration and blood collection When the first dose of arbekacin was administered, either 200 or $250 \mathrm{mg}$ was administered via intravenous infusion over $30 \mathrm{~min}$. After that, a 200- or 250-mg dose was administered via intravenous infusion over $30 \mathrm{~min}$ after hemodialysis. A polysulfone hemodialysis membrane was used for the first hemodialysis session after arbekacin administration, and AN69 was used for the second hemodialysis session. Samples were collected $30 \mathrm{~min}$ after the first

\section{a) In vitro experimental device}

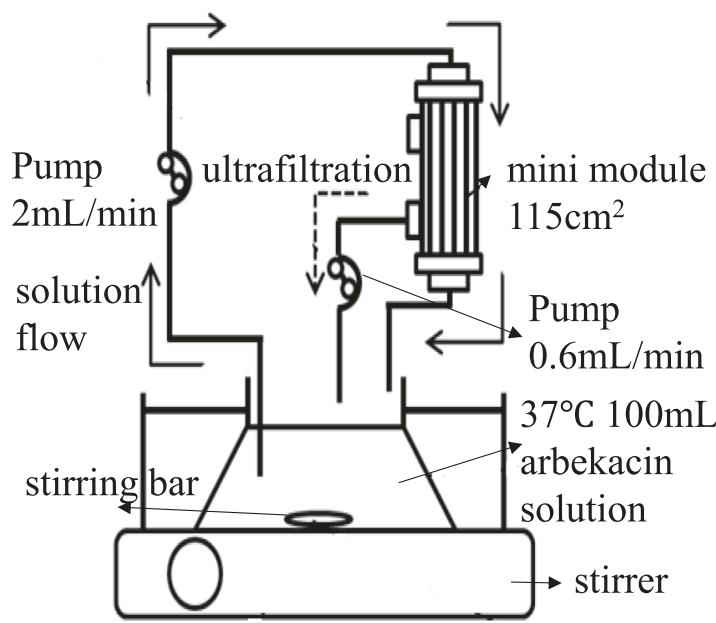

b) Changes in arbekacin concentration over time

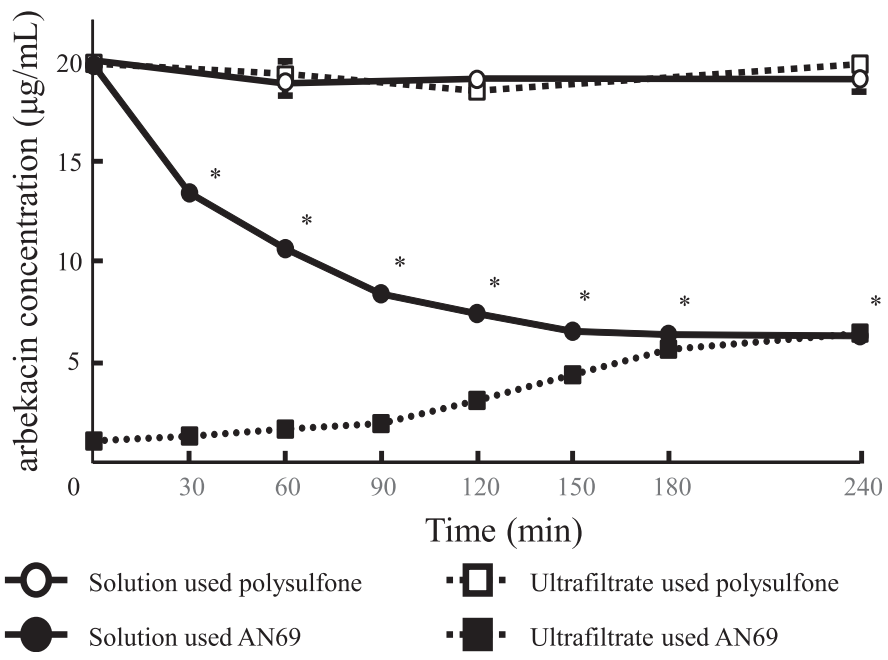

Fig. 2 Quantitative assessment of interaction of arbekacin with dialysis membranes. a In vitro experimental device. b Changes in arbekacin concentration over time. Results shown are mean \pm standard deviation $(n=3)$. ${ }^{*} P<0.05$, compared with 0 min. AN69 sulfonated polyacrylonitrile 
dose of arbekacin was administered (C1), at the start of hemodialysis with polysulfone (C2), $30 \mathrm{~min}$ after the second dose of arbekacin was administered (C5), at the start of hemodialysis with AN69 (C6), $1 \mathrm{~h}$ after the start of hemodialysis (C9), $2 \mathrm{~h}$ after the start of hemodialysis (C10), and $30 \mathrm{~min}$ after the third dose of arbekacin was administered (C11). Additionally, at 10 min after the start of hemodialysis, only blood was circulated without dialysate in order to obtain the ultrafiltrate. At that time, blood from the dialyzer inlet $(\mathrm{C} 3, \mathrm{C} 7)$ and outlet $(\mathrm{C} 4, \mathrm{C} 8)$ was collected along with the ultrafiltrate (F1, F2) (Fig. 3). Blood and ultrafiltrate were each collected as $1-\mathrm{mL}$ samples. Plasma was isolated from collected blood by centrifugation at $3000 \mathrm{rpm}$ for $10 \mathrm{~min}$. The plasma and ultrafiltrate were stored at $-80^{\circ} \mathrm{C}$ until the arbekacin concentration was measured.

4. Assessment of pharmacokinetics and removal by hemodialysis

The plasma concentration at the end of hemodialysis, the half-life $\left(t_{1 / 2}\right)$ on and off hemodialysis, and total body clearance were calculated from plasma concentrations at $\mathrm{C} 1$ through $\mathrm{C} 11$. The rate of removal by hemodialysis was calculated using the following equation.

$R=\left(C_{\text {start }}-C_{\text {end }}\right) / C_{\text {start }}$

$R$ is the rate of removal by hemodialysis (\%), $C_{\text {start }}$ is the plasma concentration at the start of hemodialysis (C2 or C6) $(\mu \mathrm{g} / \mathrm{mL})$, and $C_{\text {end }}$ is the plasma concentration at the end of hemodialysis $(\mu \mathrm{g} / \mathrm{mL})$.

To consider redistribution from tissues into the blood after hemodialysis, $C_{\text {end }}$ was calculated using the following equation.

$$
C_{\text {end }}=C_{\text {peak }}^{\prime}-C_{\text {peak }}
$$

$C_{\text {peak }}$ is the peak plasma concentration at the first dose calculated from $\mathrm{C} 1$ and $\mathrm{C} 2$ and $C_{\text {peak }}$ is the peak plasma concentration at the second or third dose calculated using the elimination rate constant calculated from $\mathrm{C} 1$ and $\mathrm{C} 2$.

However, as patient 2 was on dialysis for $5 \mathrm{~h}$, the concentration calculated at $4 \mathrm{~h}$ after the start of hemodialysis was used as $C_{\text {end }}$ in this patient. Clearance of arbekacin by the dialyzer and the sieving coefficient (SC) at $10 \mathrm{~min}$ after the start of hemodialysis were calculated using the following equations.

$$
\begin{aligned}
& \text { Arbekacin clearance by the dialyzer } \\
& \begin{aligned}
& =\left(C_{\text {int }}-C_{\text {out }}\right) / C_{\text {int }} \times \mathrm{QB} \\
\mathrm{SC} & =C_{\mathrm{f}} / C_{\text {int }}
\end{aligned}
\end{aligned}
$$

$C_{\text {int }}$ is the plasma concentration at the dialyzer inlet ( $\mathrm{C} 3$ or $\mathrm{C7}$ ), $C_{\text {out }}$ is the plasma concentration at the dialyzer outlet (C4 or C8), QB is the blood flow rate, and $C_{\mathrm{f}}$ is the concentration in the ultrafiltrate (F1 or F2).

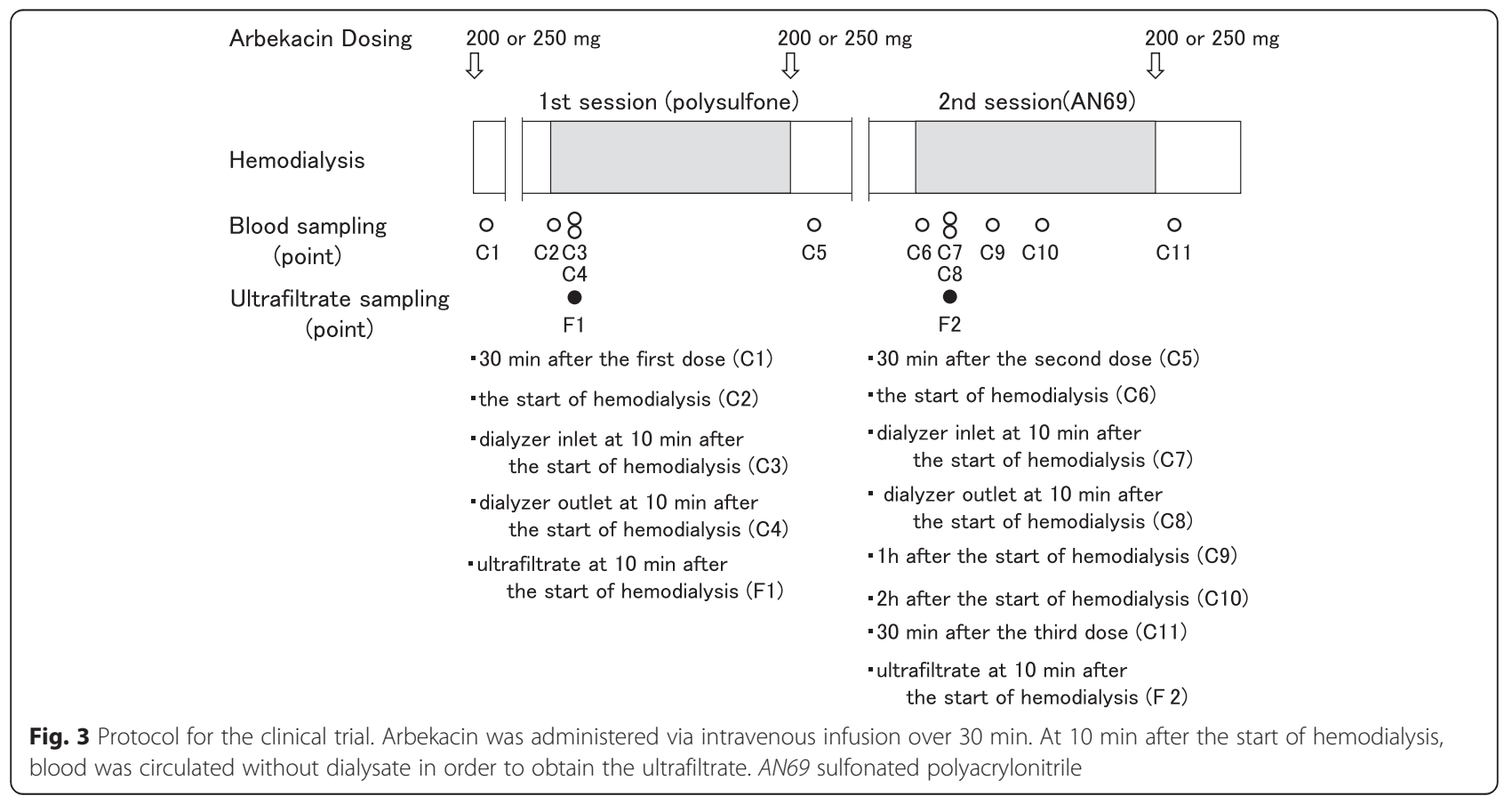




\section{Measurement of arbekacin concentration}

Arbekacin concentration was measured by fluorescence polarization immunoassay using TDX (Abbott Japan Co., Ltd., Chiba, Japan) [13].

\section{Statistical analysis}

Numbers shown are mean \pm standard deviation. Microsoft Excel 2013 was used for statistical analysis. The unpaired Student's $t$ test was used for the adsorption experiment using cut hemodialysis membranes, and the paired $t$ test was used for the in vitro adsorption experiment using a mini-module. A result of $p<0.05$ was considered statistically significant.

\section{Results}

Qualitative assessment of adsorption using cut hemodialysis membranes

The rates of arbekacin adsorption onto polysulfone and AN69 were $6.2 \pm 2.9$ and $49.8 \pm 1.8 \%$, respectively, with the rate of arbekacin adsorption onto AN69 being significantly higher (Fig. 1b).

\section{Quantitative assessment of in vitro adsorption using a mini-module}

When polysulfone was used, there was almost no change in the circulating arbekacin concentration or the arbekacin concentration in the ultrafiltrate, and the adsorption rate after 240 min was approximately $0.2 \%$. When AN69 was used, the circulating arbekacin concentration significantly decreased from $30 \mathrm{~min}$ after the start of the experiment and the adsorption rate after $240 \mathrm{~min}$ was approximately $68 \%$. The concentration of arbekacin in the ultrafiltrate gradually increased and was nearly the same as the circulating arbekacin concentration after 180 min (Fig. 2b).

\section{Clinical study}

Characteristics and hemodialysis parameters of the three patients included in the study (two men and one woman) are shown in Table 1. Changes in plasma arbekacin concentration during hemodialysis when AN69 was used are shown in Fig. 4, and pharmacokinetic parameters for each patient are shown in Table 2. For every patient, clearance was higher and $t_{1 / 2}$ was shorter on hemodialysis compared with off hemodialysis. Although clearance markedly increased and $t_{1 / 2}$ markedly decreased for up to $2 \mathrm{~h}$ from the start of dialysis when AN69 was used, clearance was lower and $t_{1 / 2}$ was higher from $2 \mathrm{~h}$ onward compared with values when patients were on hemodialysis with polysulfone. Ultimately, the rates of arbekacin removal by hemodialysis for polysulfone and AN69 were $66.7 \pm 27.3$ and $66.4 \pm 21.6 \%$, respectively, with no significant difference between them. Arbekacin concentrations in the inlet, the outlet, and the ultrafiltrate at $10 \mathrm{~min}$ after the start of dialysis when no dialysate was circulated are shown in Fig. 5. There was little difference between the arbekacin concentrations in the inlet, the outlet, and the ultrafiltrate when polysulfone was used. In contrast, the arbekacin concentrations in the outlet and the ultrafiltrate were markedly lower than that in the inlet for all patients when AN69 was used. SC for polysulfone and AN69 were $0.86 \pm 0.09$ and $0.14 \pm 0.07$, respectively.

\section{Discussion}

Arbekacin is removed by hemodialysis because it has a low molecular weight of $553 \mathrm{kDa}$, shows only 3-12\% protein binding, and has a small volume of distribution ranging from 0.2 to $0.3 \mathrm{~L} / \mathrm{kg}$. Therefore, it is believed that removal of arbekacin by hemodialysis must be taken into account when determining dosages for patients on hemodialysis [5, 6]. Although adsorption onto hemodialysis membranes has been reported as a mechanism of drug removal by dialysis [8-10], there have been very few studies

Table 1 Patient characteristics and hemodialysis parameters

\begin{tabular}{|c|c|c|c|c|c|c|c|}
\hline \multicolumn{2}{|l|}{ Case } & \multicolumn{2}{|l|}{1} & \multicolumn{2}{|l|}{2} & \multicolumn{2}{|l|}{3} \\
\hline \multicolumn{2}{|l|}{ Gender } & \multicolumn{2}{|l|}{$\mathrm{F}$} & \multicolumn{2}{|l|}{ M } & \multicolumn{2}{|l|}{ M } \\
\hline \multicolumn{2}{|c|}{ Age (year) } & \multicolumn{2}{|l|}{63} & \multicolumn{2}{|l|}{63} & \multicolumn{2}{|l|}{44} \\
\hline \multicolumn{2}{|c|}{ HD duration (month) } & \multicolumn{2}{|l|}{48} & \multicolumn{2}{|l|}{138} & \multicolumn{2}{|l|}{9} \\
\hline \multicolumn{2}{|l|}{ DW (kg) } & \multicolumn{2}{|l|}{36.0} & \multicolumn{2}{|l|}{59.8} & \multicolumn{2}{|l|}{83.3} \\
\hline \multicolumn{2}{|c|}{ Dose of arbekacin (mg) } & \multicolumn{2}{|l|}{200} & \multicolumn{2}{|l|}{250} & \multicolumn{2}{|l|}{200} \\
\hline \multicolumn{2}{|c|}{ Diagnosis } & \multicolumn{2}{|l|}{ VAl } & \multicolumn{2}{|c|}{ Subcutaneous abscess } & \multicolumn{2}{|l|}{ VAl } \\
\hline \multirow[t]{2}{*}{ Dialyzer } & Material & Polysulfone & AN69 & Polysulfone & AN69 & Polysulfone & AN69 \\
\hline & Area $\left(m^{2}\right)$ & 1.8 & 1.53 & 2.1 & 1.53 & 1.5 & 4.53 \\
\hline \multicolumn{2}{|c|}{ Blood flow rate (mL/min) } & \multicolumn{2}{|l|}{200} & \multicolumn{2}{|l|}{230} & \multicolumn{2}{|l|}{18} \\
\hline \multicolumn{2}{|c|}{ Dialysate flow rate (mL/min) } & \multicolumn{2}{|l|}{500} & \multicolumn{2}{|l|}{500} & \multicolumn{2}{|l|}{500} \\
\hline \multicolumn{2}{|c|}{ Dialysis time (min) } & \multicolumn{2}{|l|}{240} & 300 & & 240 & \\
\hline
\end{tabular}

$F$ female, $M$ male, $H D$ hemodialysis, $D W$ dry weight, $V A$ l vascular access infection, AN69 sulfonated polyacrylonitrile 


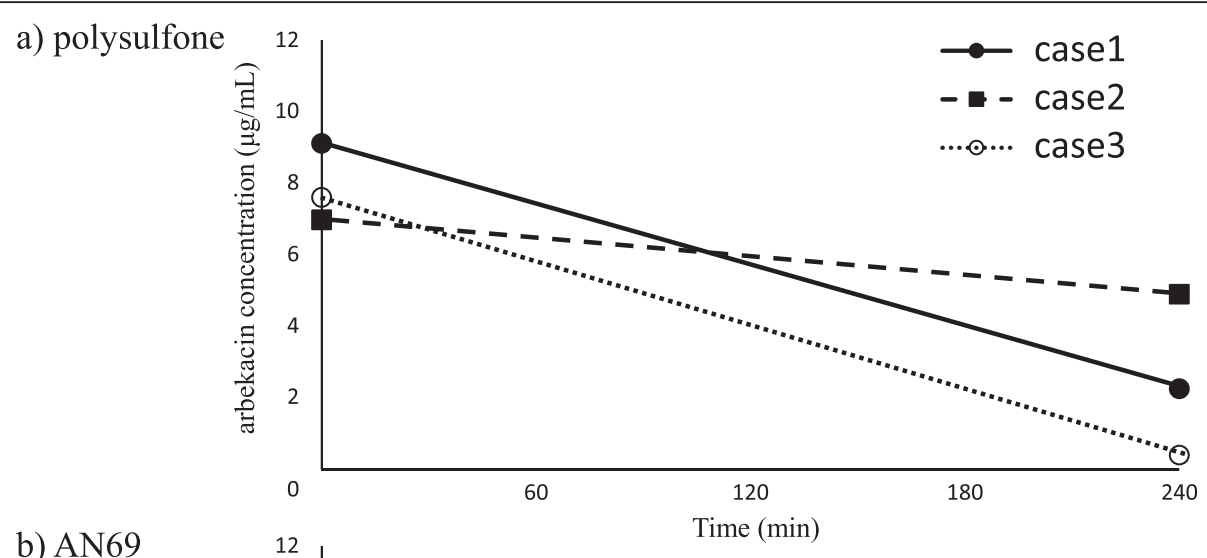

b) AN69

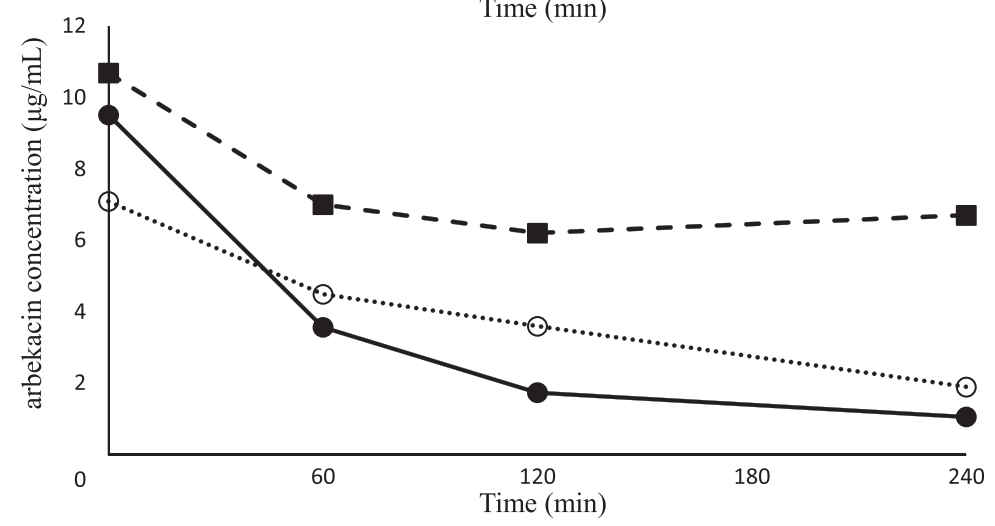

Fig. 4 Changes in plasma arbekacin concentration during hemodialysis. a Polysulfone. b AN69. AN69 sulfonated polyacrylonitrile

Table 2 Pharmacokinetic parameters of arbekacin in study patients

\begin{tabular}{|c|c|c|c|c|c|c|}
\hline \multicolumn{4}{|l|}{ Case } & \multirow{2}{*}{$\begin{array}{l}1 \\
38\end{array}$} & \multirow{2}{*}{$\begin{array}{l}2 \\
48\end{array}$} & \multirow{2}{*}{$\frac{3}{62}$} \\
\hline$t_{1 / 2}(h)$ & Off HD & & & & & \\
\hline & & \multicolumn{2}{|l|}{ Polysulfone } & 1.8 & 7.9 & 0.9 \\
\hline & \multirow[t]{3}{*}{ On HD } & & $0-1 \mathrm{~h}$ & 0.7 & 1.6 & .15 \\
\hline & & AN69 & $1-2 \mathrm{~h}$ & 1.0 & 5.7 & 3.1 \\
\hline & & & $2-4 \mathrm{~h}$ & 2.8 & n.d. & 2.2 \\
\hline & \multirow[t]{3}{*}{ Off HD } & & & 5.2 & 3.7 & 2.5 \\
\hline & & Polysulfone & & 90 & 22 & 170 \\
\hline & & & $0-1 \mathrm{~h}$ & 235 & 105 & 101 \\
\hline & \multirow[t]{2}{*}{ On HD } & AN69 & $1-2 \mathrm{~h}$ & 172 & 30 & 50 \\
\hline & & & $2-4 \mathrm{~h}$ & 59 & n.d. & 73 \\
\hline \multirow{2}{*}{\multicolumn{3}{|c|}{$\mathrm{CL}$ of arbekacin by daialyzer (mL/min) }} & Polysulfone & 3.1 & 2.4 & 4.9 \\
\hline & & & AN69 & 186 & 195 & 156 \\
\hline \multirow{2}{*}{\multicolumn{3}{|c|}{ Sieving cofficient }} & Polysulfone & 0.99 & 0.81 & .78 \\
\hline & & & AN69 & 0.05 & 0.13 & 0.23 \\
\hline
\end{tabular}

of this with arbekacin, so knowledge of its adsorption properties would likely lead to more appropriate dosage determination. In our adsorption experiment using cut hemodialysis membranes, we found that arbekacin was only minimally adsorbed onto polysulfone but was strongly adsorbed onto AN69. AN69 membranes have a strong negative charge because their chemical structure contains sulfonyl groups, whereas polysulfone membranes have almost no electrical charge. Arbekacin is a basic drug with amino groups in its chemical structure and an acid dissociation constant of 8.1, so it is positively charged in phosphoric acid buffer solution with a $\mathrm{pH}$ of 7.4. The reason why the adsorption rate of arbekacin onto AN69 was high is likely to be the formation of ionic bonds between the positively charged arbekacin with the negatively charged AN69 membrane. This result is consistent with findings from a previous study on the influence of charge of low molecular weight proteins on adsorption onto hemodialysis membranes [14] and a study showing that positively charged nafamostat mesilate adsorbed onto AN69 membrane [9], which indicates that adsorption onto hemodialysis membranes depends on the charge of adsorbed substances.

In our in vitro adsorption experiment using a minimodule, we found that when polysulfone was used, the circulating arbekacin concentration (i.e., the concentration 


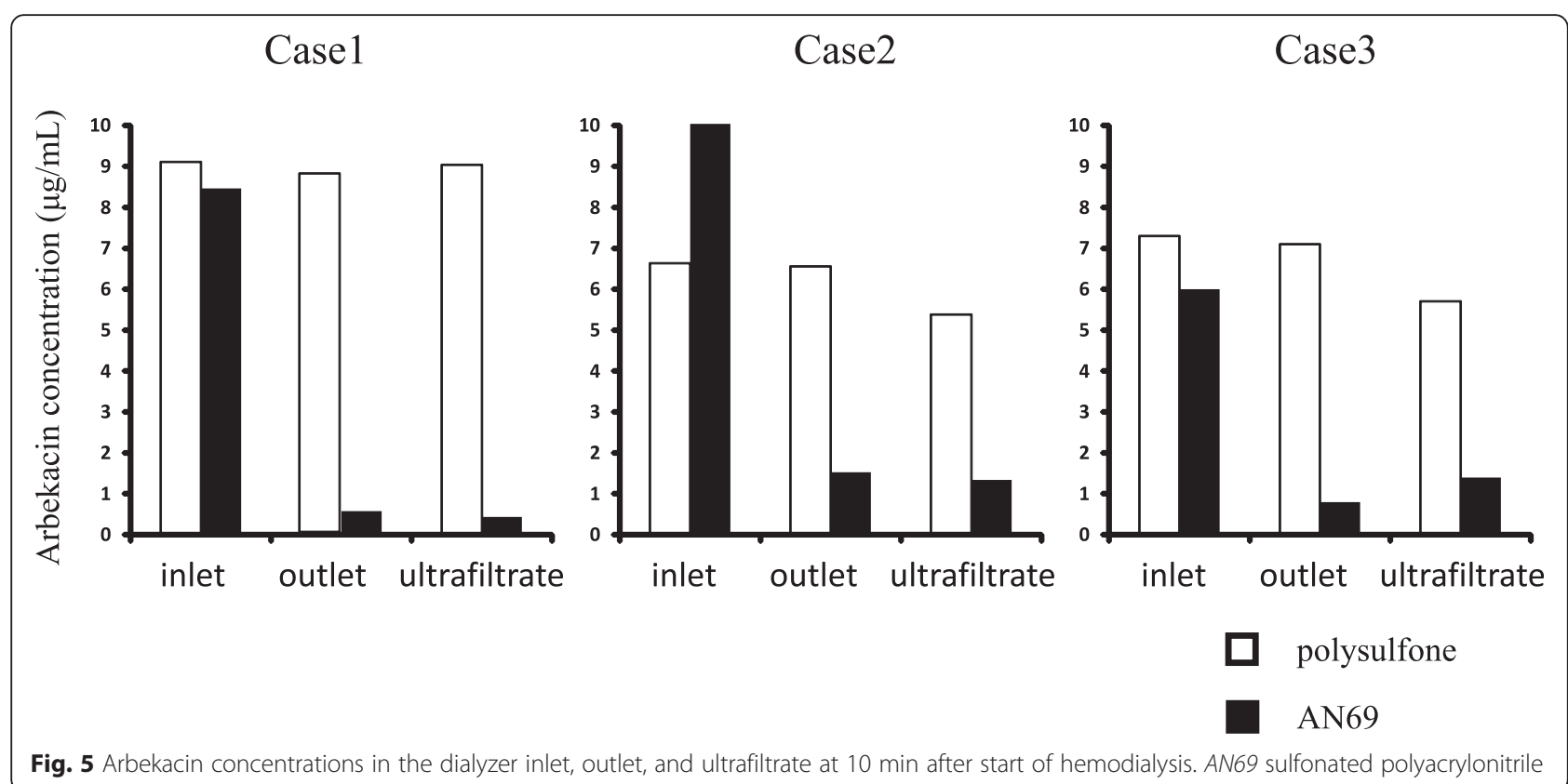

that reflects adsorption) barely changed at all over $240 \mathrm{~min}$ and the arbekacin concentration in the ultrafiltrate was the same as the circulating arbekacin concentration, which indicates that arbekacin mostly permeated through polysulfone without being adsorbed. When AN69 was used, the circulating arbekacin concentration significantly decreased from $30 \mathrm{~min}$ after the start of the experiment and had decreased by approximately $68 \%$ after $240 \mathrm{~min}$. Furthermore, the arbekacin concentration in the ultrafiltrate remained low and barely changed at all over the $90 \mathrm{~min}$ after the start of the experiment, which confirmed that arbekacin is adsorbed onto AN69. However, the concentration of arbekacin in the ultrafiltrate gradually increased and began to closely approach the circulating arbekacin concentration from $180 \mathrm{~min}$ after the start of the experiment, which indicates that adsorption reached saturation.

In our clinical study, we found that clearance was higher and $t_{1 / 2}$ was shorter on hemodialysis compared with off hemodialysis. Similarly to previous studies [6], this suggests that arbekacin is efficiently removed through hemodialysis. Furthermore, clearance of arbekacin when dialysate was not circulated was clearly greater when AN69 was used compared with when polysulfone was used (Table 2), which suggests that each membrane has a different ability to remove arbekacin or a different mechanism of removal. SC was close to 1 when polysulfone was used, which indicates that arbekacin is removed by ultrafiltration through polysulfone. This means that arbekacin easily passed through polysulfone, indicating that arbekacin is mainly removed through diffusion when dialysate is circulated. In contrast, SC was close to 0 when AN69 was used (Table 2), indicating that there is almost no ultrafiltration of arbekacin through AN69. This is probably due to increased clearance resulting from the adsorption of arbekacin onto AN69. This finding strongly suggests that the adsorption of arbekacin onto AN69 that we observed in our in vitro experiment (Fig. 2b) also occurs in clinical practice. Clearance of arbekacin decreases, starting from $2 \mathrm{~h}$ after the start of hemodialysis when AN69 is used. We also observed adsorption saturation from the 2-h mark onward in our in vitro adsorption experiment (Fig. 2b), which indicates that adsorption saturation also occurs in clinical practice. The results of our clinical study suggest that arbekacin is adsorbed onto AN69 in clinical practice as well as in vitro.

However, the clinical trial had some limitations. The sample size was small, and blood samples were collected at different time points with polysulfone than with AN69 to minimize patient discomfort. Therefore, additional research regarding the clinical impact of the adsorption of arbekacin onto AN69 will be necessary.

Many studies in which in vitro drug adsorption experiments were conducted have been published [8, 10], but a mini-module using dialyzers used in clinical practice has not previously been used to evaluate the adsorption properties of drugs. In a previous in vitro adsorption experiment that we conducted, we similarly found that the rate of arbekacin adsorption onto AN69 was approximately $70 \%$ [11]. However, the experimental system used dialyzers used in clinical practice along with arbekacin solution dissolved in bovine serum (3 L) and was at $1 / 3$ scale, so it required a large volume of bovine serum and drug. In the present study, we found adsorption rates similar to rates from previous studies and 
removal rates similar to rates from clinical studies. This suggests that our quantitative assessment system using the "mini-module" that we created for this study is suitable. In addition, the mini-module uses low volumes of solvent and drug, making it an easy-to-use and low-cost method of assessment.

\section{Conclusions}

We confirmed that arbekacin interacts with the AN69 membrane through adsorption. In addition, we found that our in vitro experimental system using a "mini-module" that we created for this study is an easier method for assessing the adsorption of drugs onto hemodialysis membranes.

\section{Abbreviations}

AN69: sulfonated polyacrylonitrile; MRSA: methicillin-resistant Staphylococcus aureus; SC: sieving coefficient; $t_{1 / 2}$ : half-life; TDM: therapeutic drug monitoring.

\section{Funding}

This work was supported by JSPS KAKENHI Grant Number 22590136.

\section{Authors' contributions}

MU was involved in the drafting of the article, conception and design of the study, and collection and assembly of data. $\mathrm{YN}$ was involved in the analysis and interpretation of data. DK was involved in the critical revision of the article for important intellectual content. KT, IT, MF, AS, YY, and KS were involved in the collection and assembly of data. SH gave the final approval of the article and was involved in the critical revision of the article for important intellectual content. All authors read and approved the final manusript

\section{Competing interests}

The authors declare that they have no competing interests.

\section{Author details}

'Division of Clinical Pharmacology, Center for Clinical Pharmaceutical Sciences, Faculty of Pharmaceutical Sciences, Kumamoto University, 5-1 Oe-Honmachi, Chuo-ku, Kumamoto 862-0973, Japan.

${ }^{2}$ Mimata-nakamachi-ten, Sogo-Pharmacy, Sogo-Medical Co., Ltd., 3481-4 Kabayama, Kitamorokata-gun, Mimata-cho, Miyazaki 89-1901, Japan. ${ }^{3}$ Department of Pharmacy, Shin-takeo Hospital, 12628 Ohaza Tomioka Takeo-cho, Takeo 843-0024, Japan. ${ }^{4}$ Department of Pharmacy, Saiseikai Kumamoto Hospital, 5-3-1 Chikami, Minami-ku, Kumamoto 861-4193, Japan. ${ }^{5}$ Department of Clinical Engineering, Saiseikai Kumamoto Hospital, 5-3-1 Chikami, Minami-ku, Kumamoto 861-4193, Japan. ${ }^{6}$ Department of Urology and Nephrology, Saiseikai Kumamoto Hospital, 5-3-1 Chikami, Minami-ku, Kumamoto 861-4193, Japan.

Received: 19 January 2016 Accepted: 6 May 2016

Published online: 20 July 2016

\section{References}

1. Aoki Y. Bactericidal activity of arbekacin against methicillin-resistant Staphylococcus aureus. Comparison with that of vancomycin. Jpn J Antibiot. 1994:47:640-6.

2. Okada K, Kimura T, Mikamo H, Kasahara K, Seki M, Takakuraet S, et al. Clinical practice guidelines for therapeutic drug monitoring of arbekacin: a consensus review of the Japanese Society of Chemotherapy and the Japanese Society of Therapeutic Drug Monitoring. J Infect Chemother. 2014;20:1-5

3. Yamasaku F, Suzuki Y, Shinkai S, Shomura T, Komiya I. Pharmacokinetics of HBK in healthy adult volunteers. Chemotherapy. 1986;34:117-28.

4. Totsuka K, Shimizu K, Mitomi N, Niizato T, Araake M. Evaluation of once-daily administration of arbekacin. Experimental study and determination of pharmacokinetic properties in man. Jpn J Antibiot. 1994;47:676-92.
5. Fillastre JP, Leroy A, Humbert G, Moulin B, Bernadet P, Josse S. Pharmacokinetics of habekacin in patients with renal insufficiency. Antimicrob Agents Chemother. 1987;31:575-7.

6. Matsuo H, Hayashi J, Ono K, Andoh K, Andoh Y, Sano Y, et al. Administration of aminoglycosides to hemodialysis patients immediately before dialysis: a new dosing modality. Antimicrob Agents Chemother. 1997:41:2597-601.

7. Jaradat MI, Moe SM. Effect of hemodialysis membranes on beta 2-microglobulin amyloidosis. Semin Dial. 2001;14:107-12.

8. Tian Q, Gomersall CD, Ip M, Tan PE, Joynt GM, Choi GY. Adsorption of amikacin, a significant mechanism of elimination by hemofiltration. Antimicrob Agents Chemother. 2008;52:1009-13.

9. Inagaki $O$, Nishian $Y$, Iwaki $R$, Nakagawa $K$, Takamitsu $Y$, Fujita $Y$. Adsorption of nafamostat mesilate by hemodialysis membranes. Artif Organs. 1992;16:553-8.

10. Nagasaka H, Miyazaki T, Naito $H$. Drugs adsorption characteristics of various dialysis membranes. Jpn J Artif Organs. 1990;19:670-3.

11. Urata M, Hirata S, Izumi S, Furukubo T, Ota M, Fijita M, et al. Adsorption of arbekacin onto dialysis membranes in vitro. Jpn J TDM. 2004;21:273-9.

12. Takesawa S, Hidai H, Sakai K. Measurment of low molecular proteins adsorption on hemodialysis membranes. Jpn J Artif Organs. 1990;19:666-9.

13. Kumon H, Mizuno A, Nasu Y, Tsugawa M, Kishi M, Ohmori H. Pharamacokinetics of arbekacin in healthy volunteers and patients with renal insufficiency. Jpn J Antibiot. 1989;42:200-7.

14. Thomas M, Valette P, Mausset AL, Déjardin P. High molecular weight kininogen adsorption on hemodialysis membranes: influence of $\mathrm{pH}$ and relationship with contact phase activation of blood plasma. influence of pre-treatment with poly(ethyleneimine). Int J Artif Organs. 2000;23:20-6.
Submit your next manuscript to BioMed Central and we will help you at every step:

- We accept pre-submission inquiries

- Our selector tool helps you to find the most relevant journal

- We provide round the clock customer support

- Convenient online submission

- Thorough peer review

- Inclusion in PubMed and all major indexing services

- Maximum visibility for your research

Submit your manuscript at www.biomedcentral.com/submit 